\title{
Coconception, installation et évaluation de banques fourragères arbustives pour I'alimentation des vaches laitières dans l'ouest du Burkina Faso
}

\author{
Ollo Sib 1,2,3,4* Eliel González-García 3,4 \\ Valérie M.C. Bougouma-Yameogo ${ }^{2}$ \\ Mélanie Blanchard ${ }^{3,4}$ Eric Vall 1,3,4
}

\begin{abstract}
Mots-clés
Bovin laitier, fourrage, plante d'abroutissement, expérimentation au champ, alimentation des ruminants, Burkina Faso
\end{abstract}

Submitted: 21 August 2018

Accepted: 11 September 2019

Published: 24 February 2020

DOI: $10.19182 /$ remvt.31841

\begin{abstract}
Résumé
Dans les élevages laitiers de l'ouest du Burkina Faso, la production de lait est limitée par un déficit fourrager en saison sèche. Cette étude visait à évaluer la faisabilité et la potentialité des banques fourragères arbustives (BFA) pour complémenter I'alimentation des vaches laitières en saison sèche avec un fourrage de bonne valeur nutritionnelle. Un travail de coconception, d'installation et d'exploitation de BFA, composées de Leucaena leucocephala et de Morus alba, a été réalisé dans trois élevages de bovins laitiers de l'ouest du Burkina Faso. Les trois BFA ont été plantées à haute densité, avec 20000 plants.ha ${ }^{-1}$, en juillet 2016. La croissance a été rapide durant les sept premiers mois (saison des pluies et début de saison sèche). Au jour de plantation (jp) plus sept mois, $L$. leucocephala mesurait $145 \pm 11 \mathrm{~cm}$ et $M$. alba $143 \pm 72 \mathrm{~cm}$. Puis, durant la saison sèche, la croissance s'est ralentie. Les caractéristiques du sol, le manque de pluies et la faible profondeur des lits de plantation expliquaient les différences de croissance observées selon les sites expérimentaux. Les attaques de termites (BFA3) et le passage du feu (BFA1) ont affecté le développement des BFA concernées sans les anéantir. La production de biomasse sur BFA2 et BFA3, cumulée sur les trois coupes d'exploitation ( $j p+13$, jp+15 et jp+17-18 mois), a été plus élevée avec $L$. leucocephala $\left(8,2 \pm 2,6\right.$ t MS.ha- $\left.{ }^{-1}\right)$ qu'avec $M$. alba $\left(1,8 \pm 2,3\right.$ t MS.ha $\left.^{-1}\right)$. Le coût d'installation et d'exploitation de $625 \mathrm{~m}^{2}$ de BFA a été de 896188 FCFA (environ $1350 €$ ). Des solutions sont à trouver pour les rendre financièrement plus accessibles aux éleveurs.
\end{abstract}

- Comment citer cet article : Sib O., González-García E., Bougouma-Yameogo V.M.C., Blanchard M., Vall E., 2020. Codevelopment, establishment and assessment of shrub fodder banks for dairy cow feeding in Western Burkina Faso. Rev. Elev. Med. Vet. Pays Trop., 73 (1): 27-35, doi: 10.19182/remvt.31841

\section{INTRODUCTION}

Au Burkina Faso, pour nourrir les vaches en lactation, les éleveurs ont recours aux pâturages naturels, aux résidus de cultures pâturés au champ, aux pailles et fanes stockées sur l'exploitation et aux aliments concentrés (sons de céréales, graines et tourteaux de coton). Certains

\footnotetext{
1. Centre international de recherche-développement sur l'élevage en zone subhumide (Cirdes), UR Systèmes de production agro-pastoraux et environnement (USPAE), 01 BP 454, Bobo-Dioulasso 01, Burkina Faso.

2. Université Nazi Boni, Institut de développement rural, Bobo-Dioulasso, Burkina Faso.

3. CIRAD, UMR SELMET, F-34398 Montpellier, France.

4. SELMET, Univ Montpellier, CIRAD, INRA, Montpellier SupAgro, Montpellier, France.

* Auteur pour la correspondance

Tél. : +22662771937 ; email : sibollo84@yahoo.fr

(c) () $\mathrm{g}$ https://creativecommons.org/licenses/by/4.0/
}

éleveurs innovent avec la production de cultures fourragères, comme le mucuna (Mucuna deeringiana) et le niébé (Vigna unguiculata). Malgré cette diversité de ressources et ces innovations, la couverture des besoins alimentaires des vaches n'est pas assurée toute l'année avec un déficit fourrager en saison sèche (Sib et al., 2017). Avec le développement des minilaiteries et la croissance de la demande en lait, certains éleveurs souhaitent accroître leur production laitière (Corniaux et al., 2014). Ils expriment une demande d'innovation pour produire, à coût limité, des fourrages de bonne valeur nutritionnelle en plus grande quantité durant la saison sèche.

Pour produire un fourrage vert durant la saison sèche, en agriculture pluviale et sous un climat soudano-sahélien, les cultures fourragères herbacées ne sont pas la solution adaptée (Klein et al., 2014). En revanche, les ligneux fourragers cultivés présentent de nombreux avantages. En Afrique, l'importance des ligneux fourragers spontanés pour nourrir le bétail en saison sèche est bien documentée (Klein et al., 2014) de même que leur impact sur la production de lait (Franzel 
et al., 2014). Cependant, les tentatives d'introduction de plantations d'arbres à vocations fourragères en Afrique restent rares. Les arbres fourragers sont parfois retrouvés dans des dispositifs de haies de délimitation des parcelles et/ou pour fertiliser les sols, mais rarement pour alimenter les animaux (Franzel et al., 2014 ; Bationo et al., 2012)

Or, dans certaines régions tropicales d'Amérique latine, des Caraïbes et d'Asie du Sud, des systèmes fourragers agroforestiers basés sur des banques fourragères arbustives (BFA), plantations à haute densité d'arbres fourragers exploités pour l'affouragement à l'auge, ont été développés et bien adoptés par les éleveurs (Datta, 2000 ; González-García et al., 2009 ; González-García et Martín-Martín, 2016). Les BFA présentent des avantages économiques et environnementaux dans un large éventail de situations des tropiques subhumides (Franzel et al., 2014).

Dans le contexte plus sec du Burkina Faso, nous avons émis l'hypothèse que la BFA peut trouver une place dans le système fourrager pour améliorer l'alimentation des vaches en lactation durant la saison sèche, mais aussi pendant le pic de lactation (entre juillet et août). La BFA pourrait compléter les ressources disponibles, à condition de trouver des réponses aux contraintes que sont le manque de connaissances des éleveurs sur les itinéraires techniques, le stockage et l'utilisation des fourrages, le manque de disponibilité en semences fourragères, le manque de main-d'œuvre et d'espace disponible, et l'absence de matériel de fauche et de bâtiments de stockage dans les élevages (Klein et al., 2014). Dans le cas de la BFA, le droit coutumier de planter des arbres, reconnu aux seuls propriétaires terriens, la divagation des troupeaux en saison sèche et les feux de brousse sont des contraintes supplémentaires.

Pour prendre en compte ces multiples contraintes techniques et socioéconomiques inhérentes à l'introduction d'une BFA dans le système fourrager, et pour accompagner les éleveurs dans l'apprentissage de cette innovation, nous avons mis en place un dispositif de recherche en partenariat. La présente étude porte sur cette recherche d'accompagnement qui s'est basée sur une démarche de coconception, d'expérimentation et d'évaluation de la BFA chez l'éleveur (Vall et al., 2016).

\section{MATERIEL ET METHODES}

\section{Etape de coconception des BFA}

\section{Choix des sites et des volontaires pour la mise en place des BFA}

Trois sites ont été sélectionnés dans la région des Hauts-Bassins à l'ouest du Burkina Faso pour la mise en place des BFA. Le site de Sagassiamasso (BFA1 ; $11^{\circ} 20^{\prime} \mathrm{N}, 4^{\circ} 13^{\prime} \mathrm{W} ; 386 \mathrm{~m}$ d'altitude), le site de Koumbia (BFA2 ; $11^{\circ} 18^{\prime}$ N, $3^{\circ} 45^{\prime} \mathrm{W}$; 309 m d'altitude), dans la province du Tuy, et le site de Nasso (BFA3 ; $11^{\circ} 14^{\prime} \mathrm{N}, 4^{\circ} 27^{\prime} \mathrm{W}$; $386 \mathrm{~m}$ d'altitude). Dans ces trois sites, la saison des pluies s'étend de juin à octobre, avec un régime de précipitations variant entre 800 et $1000 \mathrm{~mm}$ par an. Les températures moyennes se situent entre 23 et $35^{\circ} \mathrm{C}$ et l'humidité relative entre $27 \%$ et $80 \%$.

Ces trois sites sont concernés par le développement de la production laitière, mais avec des contraintes qui diffèrent légèrement d'un site à l'autre. Les éleveurs de Sagassiamasso bénéficient de la proximité du marché urbain, mais leurs vaches laitières ont peu accès au pâturage et aux fourrages de qualité. A Koumbia, l'accès au pâturage est un problème observé pendant toutes les saisons. A Nasso, près de la forêt classée de Dinderesso, le pâturage n'est disponible qu'en saison des pluies.

Sur chaque site, des ateliers impliquant les éleveurs expérimentateurs et leurs voisins, les techniciens de l'élevage, les chefs coutumiers, les élus, et les représentants des laiteries ont été tenus en mars 2016 pour restituer les résultats du diagnostic initial, identifier des éleveurs expérimentateurs volontaires, ajuster la technologie des BFA et préparer leur installation, puis en mars 2017 pour présenter les résultats de l'installation des BFA et préparer l'étape de leur exploitation.

L'expérimentation des BFA s'est appuyée sur des éleveurs volontaires ayant un noyau de vaches laitières et disposant d'une parcelle à cultiver. Les volontaires s'engageaient à préparer le sol avant la plantation, désherber et créer un pare-feu, suivre, fertiliser, et exploiter la BFA, en accord avec les recommandations discutées avec les chercheurs lors des ateliers. Les chercheurs s'engageaient de leur côté à apporter un appui technique pour l'implantation, le suivi et l'exploitation des BFA, à fournir les plants et à construire les clôtures. Trois éleveurs répondants aux critères établis ont été sélectionnés et accompagnés dans la mise en place d'une BFA, soit un sur chacun des trois sites.

\section{Choix des espèces fourragères et dispositifs expérimentaux}

Deux espèces à usages multiples ont été choisies : Leucaena leucocephala (Wencomo et Ortiz, 2009 ; González et al., 2009) et Morus alba (Pentón et al., 2007 ; González-García et Martín-Martín, 2016), reconnues pour leur valeur nutritionnelle, leur aptitude à croître rapidement, leur productivité élevée, leur résistance à la sécheresse et à la coupe, et leur capacité d'adaptation dans divers milieux. Pour la haie vive, Gliricidia sepium, Albizia lebbeck et Samanea saman ont été retenues. Les graines ont été acquises à la station expérimentale Indio Hatuey à Cuba (M. alba, L. leucocephala et G. sepium), au Centre national de semence forestière (CNSF), et à l'Association pour la promotion des arbres fertilitaires, de l'agroforesterie et la foresterie (APAF) à Ouagadougou, Burkina Faso (A. lebbeck et S. saman).

Le schéma d'implantation des BFA a été discuté avec les éleveurs, en ayant à l'esprit qu'il s'agissait de dispositifs expérimentaux pilotes qui ne pouvaient donc pas être d'emblée dimensionnés à la hauteur des besoins réels des éleveurs expérimentateurs (figure 1). Les parcelles ont été limitées à $625 \mathrm{~m}^{2}$. Une densité de plantation de 25000 plants. $\mathrm{ha}^{-1}$ ( $1 \mathrm{~m}$ entre les lignes et $0,4 \mathrm{~m}$ entre les plants) a été proposée pour optimiser la croissance des plants en hauteur (Noda et Martín, 2008). Une disposition des deux espèces (L. leucocephala et M. alba) en

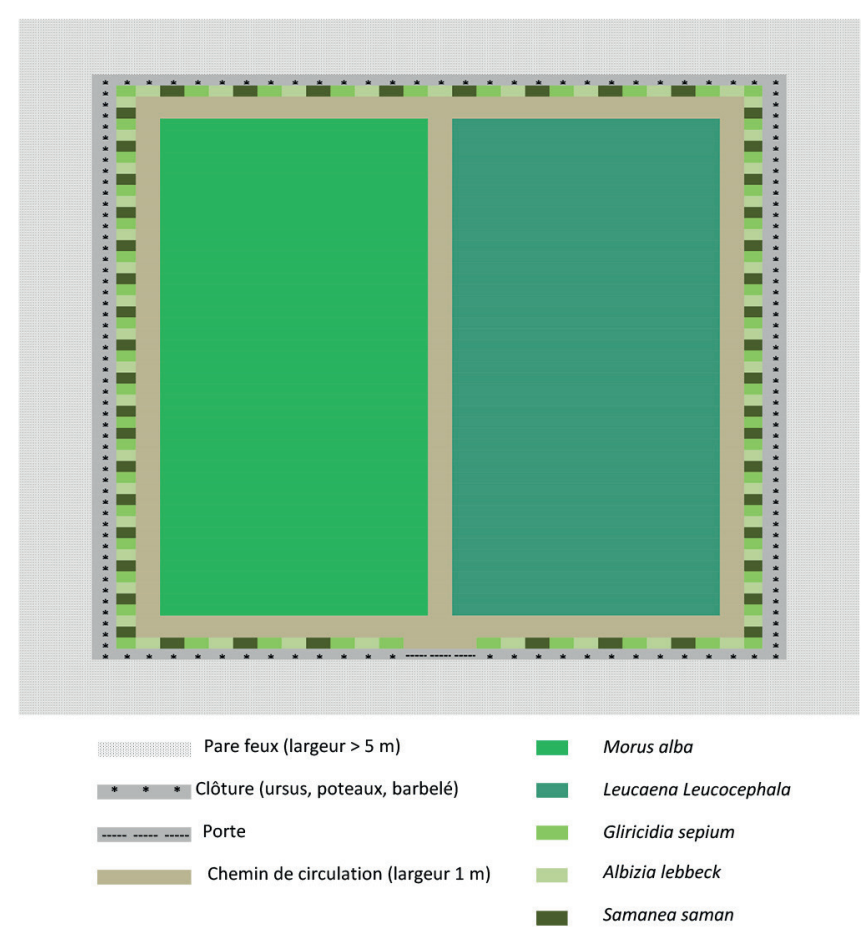

Figure 1 : schéma d'implantation d'une banque fourragère arbustive dans l'ouest du Burkina Faso. 
deux blocs distincts a été retenue afin de suivre séparément le comportement des deux espèces et de minimiser les risques en cas de maladie. Pour la protection des BFA, l'installation d'une clôture en grillage Ursus a été installée pour éviter l'intrusion d'animaux divagants dès la première année. Il a été convenu de compléter cette clôture par une haie vive de G. sepium, A. lebbeck et $S$. saman, plantés en alternance avec un écartement de $50 \mathrm{~cm}$, destinés à assurer la protection de la BFA à moyen terme pendant la durée de son exploitation.

L'emplacement des BFA a été choisi en fonction des critères de proximité a) des parcs à bétail pour réduire la durée des opérations d'affouragement à l'auge et b) d'un point d'eau pour faciliter l'arrosage des plants en cas d'une importante sécheresse. Il a été tenu compte de la proximité du domicile familial pour surveiller l'intrusion d'animaux en divagation. Les sols devaient être bien drainés pour assurer une bonne installation des plants. Des échantillons composites de sols ont été prélevés sur les horizons $0-20$ et $20-40 \mathrm{~cm}$ des parcelles, et analysés au laboratoire de l'Institut national de l'environnement et de recherches agricoles (Inera) Farako-Ba à Bobo-Dioulasso, Burkina Faso (tableau I).

\section{Gestion prévisionnelle de l'étape d'installation des BFA}

Pour l'installation des BFA, l'itinéraire technique utilisé par González-García et Martín-Martín (2016) a été suivi avec deux étapes : a) la production des pépinières (de décembre 2015 à juin 2016), puis b) l'installation proprement dite des BFA chez les éleveurs (de juillet 2016 à juin 2017).

\section{Production des plants en pépinière}

La production des plants a été confiée à un pépiniériste professionnel à Bobo-Dioulasso, parce que les éleveurs ne maîtrisaient pas encore cette technique. Toutes les graines utilisées ont bien germé sauf celles de M. alba due à la qualité moyenne des graines. Au total, 1820 plants de L. leucocephala de $47 \pm 25 \mathrm{~cm}$ de hauteur et 1620 plants de $M$. alba de $38 \pm 18 \mathrm{~cm}$ de hauteur ont été produits. Ainsi, 1200, 1100 et 1140 plants des deux espèces ont été plantés, respectivement sur les BFA1, BFA2 et BFA3.

\section{Plantation et suivi de l'installation des BFA}

Pour préparer l'installation, il a été convenu avec les éleveurs de réaliser un épandage de fumier de parc bovin à une dose de 2,5 tonnes de matière sèche (MS) par hectare. Deux labours croisés en traction attelée ont été réalisés, le premier au moment des premières pluies et le deuxième quelques jours avant la plantation. Des sillons de $30 \mathrm{~cm}$ de profondeur et de $20 \mathrm{~cm}$ de diamètre ont été prévus pour recevoir les jeunes plants. Les BFA ont été plantées au début de la saison des pluies, entre fin juin et début juillet.

Globalement, les dispositions retenues lors des réunions de préparation avec les éleveurs et précisées dans les cahiers des charges ont été respectées. Le faible taux de germination des graines de $M$. alba a entraîné un ajustement de la densité de plantation à la quantité de plants disponibles, soit 20000 plants.ha $^{-1}$ au lieu de 25000 , comme initialement prévu.

\section{Suivi de l'installation des BFA et analyses statistiques}

Dans chacune des BFA, $10 \%$ de plants de L. leucocephala et $10 \%$ de plants de $M$. alba ont été suivis une fois par mois pendant l'étape d'installation (de juillet 2016 à juin 2017). Les mesures ont porté sur la hauteur des plants $(\mathrm{cm})$, la longueur des branches $(\mathrm{cm})$, le nombre de branches et le diamètre de la tige $(\mathrm{mm})$. Les mortalités, attaques des termites, maladies et autres événements (feux, etc.) ont été enregistrés à chaque passage. Les données mesurées ont été transcrites dans une base de données sous Excel 2010. Dans cet article, seuls les résultats concernant la hauteur des plants (en $\mathrm{cm})$, bien corrélée à la biomasse disponible (Chave et al., 2015), sont présentés.

Des analyses de variance (Anova) à deux facteurs ont été réalisées à la fin de chaque étape durant la période d'installation : à la fin de la saison des pluies (octobre, jour de plantation [jp]+4 mois) et à la fin de la saison sèche froide (janvier, $\mathrm{jp}+7$ mois), afin de comparer les performances de croissance des BFA. Dans l'Anova les variables explicatives (effets fixes) étaient le site d'implantation de la BFA et la saison (mesurée en mois de l'année) ; la variable à expliquer était la hauteur des plants. L'équation de l'Anova (XLSTAT 2018.1.49131 ; www.xlstat.com/fr) a été définie comme suit :

Yijk $=\mu+$ Site $\mathrm{i}+$ Date $\mathrm{j}+($ Site $\times$ Date $) \mathrm{ij}+$ cijk

où Yijk est la variable mesurée à la date $\mathrm{j}$ sur le site $\mathrm{i}, \mu$ la moyenne globale, Site i l'effet fixe du site expérimental i (i $=1-3)$, Date j l'effet fixe de la date de la saison de mesure $\mathrm{j}(\mathrm{j}=1-12)$, (Site $\times$ Date) $\mathrm{ij}$ l'effet fixe de l'interaction entre l'effet du site expérimental i et l'effet de la date des saisons de mesure j, et eijk l'erreur résiduelle ou erreur expérimentale.

\section{Gestion prévisionnelle de l'étape d'exploitation des BFA}

Létude de la production de biomasse fourragère n'a concerné que les BFA2 et BFA3, car la BFA1 a été dévastée par un incendie à jp+8 mois. En théorie, la fin de l'étape d'installation de la BFA devait

\section{Tableau}

Caractéristiques des sols des parcelles d'implantation des banques fourragères arbustives (BFA) dans trois localités de l'ouest du Burkina Faso

\begin{tabular}{|c|c|c|c|c|c|c|c|c|c|c|}
\hline \multirow[b]{2}{*}{ Site } & \multirow[b]{2}{*}{$\begin{array}{c}\text { Horizon } \\
(\mathrm{cm})\end{array}$} & \multicolumn{6}{|c|}{ Paramètre chimique } & \multicolumn{3}{|c|}{ Texture (\%) } \\
\hline & & $\mathrm{pH}$ & $\begin{array}{l}\text { MO } \\
(\%)\end{array}$ & $\begin{array}{c}\mathrm{C} \\
(\%)\end{array}$ & $\begin{array}{c}N \\
(\%)\end{array}$ & $\mathrm{C} / \mathrm{N}$ & $\begin{array}{c}\mathrm{P}^{*} \\
(\mathrm{mg} / \mathrm{kg} \text { sol) }\end{array}$ & Argile & Limon & Sable \\
\hline \multirow[t]{2}{*}{ BFA1 } & 0-20 & 6,3 & 0,5 & 0,3 & 0,03 & 11 & 7,2 & 7,9 & 8,2 & 83,8 \\
\hline & $20-40$ & 5,7 & 0,3 & 0,2 & 0,02 & 12 & 2,0 & 14,8 & 7,2 & 77,8 \\
\hline \multirow[t]{2}{*}{ BFA2 } & $0-20$ & 6,1 & 0,6 & 0,3 & 0,03 & 12 & 6,6 & 6,9 & 12,2 & 80,8 \\
\hline & $20-40$ & 6,7 & 0,3 & 0,2 & 0,02 & 10 & 1,9 & 11,8 & 11,2 & 76,8 \\
\hline \multirow[t]{2}{*}{ BFA3 } & $0-20$ & 5,3 & 0,7 & 0,4 & 0,03 & 13 & 3,8 & 21,5 & 23,5 & 54,9 \\
\hline & $20-40$ & 5,1 & 0,4 & 0,2 & 0,03 & 9 & 2,0 & 27,4 & 19,6 & 52,9 \\
\hline
\end{tabular}

MO : matière organique ; $\mathrm{C}$ : carbone $; \mathrm{N}$ : azote $; \mathrm{P}^{*}$ : phosphore assimilable 
intervenir 12 mois après la plantation. Pour l'étape d'exploitation, une coupe d'uniformisation à $50 \mathrm{~cm}$ de hauteur suivie de deux récoltes de fourrages avait été décidée. Cependant, les observations à 12 mois ont montré que les plants n'étaient pas encore aptes à supporter une coupe trop basse. Ainsi, la coupe d'uniformisation a été réalisée à la hauteur de $120 \mathrm{~cm}$ sur la totalité des plants à jp+13 mois. Une deuxième coupe a été faite à jp+15 mois sur tous les plants en une fois, à la hauteur de $50 \mathrm{~cm}$. Entre jp+17 et jp+18 mois, la troisième coupe a été réalisée quotidiennement et destinée à complémenter des vaches à la traite en affouragement à l'auge. Elle a été réalisée sur 17 pieds par jour de L. leucocephala et 14 pieds par jour de M. alba dans la BFA2, et sur 14 pieds par jour de L. leucocephala et six pieds par jour de M. alba dans la BFA3. Pour que les BFA soient exploitables après la coupe d'uniformisation, la condition était que les rejets des plants aient une longueur supérieure ou égale à $50 \mathrm{~cm}$.

La biomasse récoltée à chaque coupe était pesée puis un échantillon était mis à l'étuve $\left(60^{\circ} \mathrm{C}\right.$ pendant 48 heures) pour déterminer le taux de matière sèche. Les rendements moyens étaient alors calculés comme suit :

rendement MS.plant ${ }^{-1}=$ (poids fourrage frais / nb. de pieds coupés $) \times$ $\% \mathrm{MS}$

rendement $\mathrm{MS} \cdot \mathrm{bloc}^{-1}=$ poids fourrage frais d'un bloc $\times \% \mathrm{MS}$ rendement MS.hat ${ }^{-1}=$ rendement MS.bloc ${ }^{-1} \times$ nb. de blocs.ha ${ }^{-1}$

Après détermination de la MS du fourrage frais, la composition chimique des fourrages récoltés a été prédite par spectroscopie dans le proche infrarouge (SPIR) au laboratoire d'analyse des aliments fourragers du Cirad à Montpellier. Les composants prédits étaient : la MS, la matière minérale (MM), les matières azotées totales (MAT), les constituants pariétaux selon la méthode de Van Soest (NDF, ADF) et la lignine (ADL), la cellulose brute (CB), la solubilité de la matière sèche (SMS), et la solubilité de la matière organique (SMO). Pour comparer les rendements et les principaux constituants chimiques (MAT, NDF, ADF) des deux BFA (BFA2 et BFA3) entre les deux espèces et entre les sites, le test de Kruskal-Wallis et la comparaison multiple par paire suivant la procédure de Steel-Dwass-CritchlowFligner ont également été réalisés sur XLSTAT 2018.

\section{RESULTATS}

\section{Résultats de l'étape d'installation}

\section{Ajustement de l'installation des BFA}

La période de plantation idéale a coïncidé avec la période de mise en place des autres cultures dans les exploitations (maïs, coton, etc.) durant laquelle les éleveurs renforçaient le gardiennage des animaux pour éviter tout dommage sur les cultures fraîchement semées. Ceci explique pourquoi les éleveurs expérimentateurs n'ont pas été disponibles pour l'installation des BFA avant la fin du mois de juin. L'écart maximum d'une semaine prévu entre l'implantation des trois BFA a été respecté à peu de jours près entre la BFA1 implantée le 24 juin, la BFA2 le 3 juillet et la BFA3 le 10 juillet (tableau II).

Dans les trois BFA, les lits de plantation ont été ajustés avec des profondeurs parfois inférieures aux $30 \mathrm{~cm}$ initialement prévus en raison du manque de main-d'œuvre chez certains expérimentateurs (BFA1, $30 \mathrm{~cm}$; BFA2, $20 \mathrm{~cm}$; BFA3, $20 \mathrm{~cm}$ ). La fumure organique (FO) a été initialement apportée à la dose de 2,5 $\mathrm{t} \mathrm{MS}$.ha ${ }^{-1}$ par BFA. Dans les BFA1 et BFA2, la dose de paillage a été de 5,0 t MS.ha ${ }^{-1}$ au lieu de 10,0 t MS.ha ${ }^{-1}$ prévues, et dans la BFA3 le paillage prévu a été abandonné, faute de main-d'œuvre.

\section{Performance de croissance des ligneux}

Les plants ont suivi un développement soutenu jusqu’à la fin de la saison des pluies à jp+4 mois. Durant la saison sèche froide, de jp+5 à jp+7 mois, la croissance des BFA s'est poursuivie à un rythme moins rapide.

Au terme des deux premières étapes de croissance des plants, à jp+4 et à jp+7 mois, l'analyse statistique a montré des différences

\section{Tableau II}

Itinéraire technique et suivi des performances des banques fourragères arbustives (BFA) dans trois localités de l'ouest du Burkina Faso

\begin{tabular}{|c|c|c|c|c|c|c|}
\hline & \multicolumn{3}{|c|}{ Leucaena leucocephala } & \multicolumn{3}{|c|}{ Morus alba } \\
\hline & BFA1 & BFA2 & BFA3 & BFA1 & BFA2 & BFA3 \\
\hline \multicolumn{7}{|l|}{ Etape de plantation } \\
\hline Date de plantation & 24 juin & 3 juillet & 10 juillet & 24 juin & 3 juillet & 10 juillet \\
\hline Densité de plantation (plants.ha ${ }^{-1}$ ) & & & 20 & & & \\
\hline Dose de fumure organique (t MS.ha ${ }^{-1}$ ) & 1,2 & 1,2 & 1,2 & 1,2 & 1,2 & 1,2 \\
\hline Dose de paillage (t MS.ha- ${ }^{-1}$ ) & 5,0 & 5,0 & 0,0 & 5,0 & 5,0 & 0,0 \\
\hline \multicolumn{7}{|l|}{ Etape d'installation } \\
\hline Hauteur à jp (cm) & $50 \pm 24$ & $43 \pm 26$ & $50 \pm 25$ & $31 \pm 17$ & $42 \pm 19$ & $33 \pm 17$ \\
\hline Hauteur à jp+4 mois fin sdp (cm) & $146 \pm 59^{a}$ & $145 \pm 42^{\mathrm{a}}$ & $93 \pm 32^{b}$ & $156 \pm 52^{\mathrm{a}}$ & $121 \pm 32^{b}$ & $113 \pm 38^{b}$ \\
\hline Hauteur à jp+7 mois fin ssf (cm) & $165 \pm 60^{\mathrm{a}}$ & $160 \pm 40^{\mathrm{a}}$ & $109 \pm 33^{b}$ & $175 \pm 47^{\mathrm{a}}$ & $127 \pm 33^{b}$ & $128 \pm 37^{b}$ \\
\hline Mortalité termites (\%) & 6 & 5 & 25 & 3 & 19 & 64 \\
\hline \multicolumn{7}{|l|}{ Etape d'exploitation } \\
\hline Rendement CU à jp+13 mois (t MS.ha $\left.{ }^{-1}\right)$ & AbsD & $2,4^{\mathrm{a}}$ & $2,2^{\mathrm{a}}$ & AbsD & $0,4^{b}$ & $0,06^{\mathrm{C}}$ \\
\hline Rendement C2 à jp+15 mois (t MS.ha-1) & AbsD & $5,7^{\mathrm{a}}$ & $3,7^{\mathrm{a}}$ & AbsD & $2^{b}$ & $0,3^{\mathrm{c}}$ \\
\hline Rendement C3 à jp+18 mois (t MS.ha ${ }^{-1}$ ) & AbsD & $1,4^{\mathrm{a}}$ & $1,0^{\mathrm{a}}$ & $\mathrm{AbsD}$ & $0,6^{b}$ & $0,3^{b}$ \\
\hline Rendement cumulé (t.MS.ha-1) & $\mathrm{AbsD}$ & 9,5 & 6,9 & AbsD & 3,0 & 0,7 \\
\hline
\end{tabular}

jp : jour de plantation ; $\mathrm{CU}$ : coupe d'uniformisation ; $\mathrm{C} 2, \mathrm{C} 3$ : coupes 2 et 3 ; sdp : saison des pluies ; ssf : saison sèche froide ; ssc : saison sèche chaude ; AbsD : absence de données ; ${ }^{\mathrm{a}, \mathrm{b}, \mathrm{c}}$ Les valeurs suivies de la même lettre sur la même ligne ne sont pas significativement différentes au seuil de $5 \%$. 
significatives $(\mathrm{p}<0,0001)$ des hauteurs dans les trois BFA. A jp+4 mois, L. leucocephala a présenté des plants de hauteur similaire dans les BFA1 et BFA2, et de hauteur inférieure dans la BFA3; $M$. alba a présenté des plants de hauteur similaire dans les BFA2 et BFA3, et de hauteur supérieure dans la BFA1. Ce constat a été le même à jp+7 mois au niveau des deux espèces dans les trois BFA.

Durant la saison sèche chaude, de jp+8 à jp+10 mois, la croissance a ralenti et s'est quasi arrêtée, ce qui s'est traduit par un plateau de la courbe de croissance dans les BFA2 et BFA3. Avec le retour des pluies, à partir de jp+11 mois, la croissance des ligneux est repartie (figure 2).

\section{Facteurs limitants}

Un premier facteur limitant a concerné la pauvreté des sols en matière organique $(0,5-0,7 \%$ et $0,3-0,4 \%$, respectivement sur les horizons $0-20 \mathrm{~cm}$ et $20-40 \mathrm{~cm})$ et en phosphore assimilable $(3,8-7,2$ mg.kg-1 de sol et 1,9-2 mg.kg-1 de sol, respectivement sur les horizons 0-20 $\mathrm{cm}$ et 20-40 cm). De plus, le sol de la BFA3 avait un pH plus acide (5,3 sur l'horizon $0-20 \mathrm{~cm})$. Les sols étaient en majorité à dominance sableuse avec un pourcentage de limon et d'argile plus élevé dans la BFA3 (tableau I).

Les BFA n'ont pas subi d'intrusion d'animaux ruminants car les dispositifs de protection ont fonctionné. Par contre, elles ont souffert d'attaques de termites qui ont constitué le deuxième facteur limitant. Les premières attaques de termites sont survenues au stade juvénile des BFA durant la saison des pluies, puis se sont répétées durant la saison sèche chaude. Au cours de la saison des pluies, les attaques de termites ont eu peu d'effets sur la mortalité des jeunes plants grâce à l'usage de l'insecticide carbofuran (C12H15NO3). Les attaques les plus destructrices ont eu lieu à partir de jp+8 mois, au début de la saison sèche chaude. La BFA2 qui était paillée a subi des attaques de termites moins fortes $(4,5 \%$ et $19 \%)$ que la BFA3 non paillée ( $25 \%$ et $64 \%$ ), respectivement de mortalités de L. leucocephala et de M. alba (figure 2).

Un dernier facteur limitant était le risque d'incendie du paillage des BFA. Malgré le pare-feu installé autour de la BFA1, un feu de brousse a enflammé le paillis de la BFA1 à jp+8 mois affectant fortement les plants. Néanmoins, le passage du feu n’a pas anéanti la BFA. Un
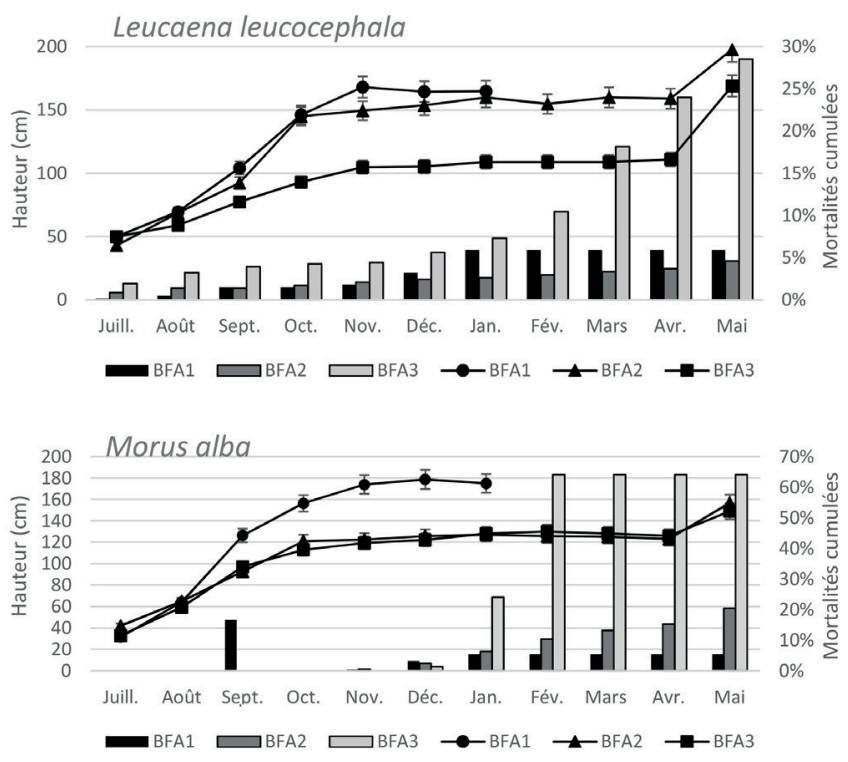

Figure 2 : évolution de la hauteur des plants (courbes) et de la mortalité mensuelle cumulée (histogrammes) de Leucaena leucocephala et de Morus alba sur des banques fourragères arbustives (BFA) dans l'ouest du Burkina Faso de juillet 2016 à mai 2017. dispositif d'irrigation hebdomadaire a été mis en place et un paillage systématique de la BFA a été réalisé pour assurer la régénération des plants. La régénération des plants a été suivie mensuellement jusqu’à la saison des pluies à jp+13 mois. Ce suivi a montré une régénération de $50 \%$ des plants de $M$. alba à jp+13 mois, un regain plus élevé que pour les plants de L. leucocephala (20\% de régénération à la même date). Les repousses de L. leucocephala ont également subi une attaque de termites à jp+12 mois, ce qui a entraîné des mortalités supplémentaires et a expliqué une régénération plus faible. A jp+13 mois, la BFA1 comptait 300 plants de M. alba régénérés et 300 autres replantés, et 120 plants de L. leucocephala régénérés et 480 jeunes plants replantés. A cette date, la BFA1 n'était donc pas exploitable. Les résultats de l'étape d'exploitation des BFA n'ont ainsi porté que sur les BFA2 et BFA3.

\section{Résultats de l'étape d'exploitation des BFA}

\section{Ajustements de l'exploitation des BFA}

Une coupe d'uniformisation a été réalisée sur les BFA2 et BFA3 à jp+13 mois à la hauteur de $120 \mathrm{~cm}$ au lieu de $50 \mathrm{~cm}$ comme initialement prévu. Elle avait pour but de rajeunir les plants et de les forcer à produire de nouveaux rejets.

Une deuxième coupe correspondant à une coupe d'uniformisation a été réalisée à jp+15 mois à la hauteur de $50 \mathrm{~cm}$. Elle avait pour but de rajeunir une fois de plus la BFA, et de préparer les plants à supporter les coupes quotidiennes à venir pour nourrir les animaux.

La troisième coupe (première récolte proprement dite) a été réalisée de jp+17 à jp+18 mois. Une période de 30 jours avait été programmée afin de simuler une exploitation réelle de la BFA en vue de la complémentation de vaches en lactation et pour étudier la qualité du fourrage pendant la période de récolte.

\section{Production de biomasse et composition chimique du fourrage}

Les rendements moyens cumulés calculés sur les BFA2 et BFA3 sur les trois périodes de coupes (jp+13,jp+15 et jp+17-18 mois) étaient de $8,2 \pm 2,6 \mathrm{t} \mathrm{MS} . h^{-1}$ pour L. leucocephala, et de 1,8 $\pm 2,3 \mathrm{t} \mathrm{MS.ha}^{-1}$

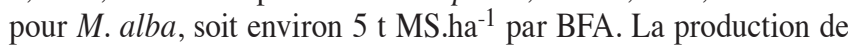
biomasse a augmenté de jp+13 à jp+15 mois et a régressé à jp+17-18 mois (tableau II).

Le rendement de L. leucocephala a été plus élevé que celui de M. alba aux trois périodes de coupes. Entre les sites, le rendement de M. alba de la BFA2 a été significativement plus élevé que celui de la BFA3 $(p=0,05)$. En revanche, il n'y a pas eu de différence significative entre les rendements de L. leucocephala sur les deux BFA.

L. leucocephala avait à jp+13 et jp+15 mois des teneurs plus élevées en constituants pariétaux (NDF et ADF) et en MAT que M. alba. Cependant, à jp+17 et jp+18 mois les compositions chimiques des deux espèces dans les deux sites ont été similaires. La teneur en MAT a évolué inversement à la teneur en NDF et ADF en fonction des périodes de coupes (tableau III). La distribution des fourrages verts aux vaches a montré une rapide adaptation de celles-ci aux fourrages des BFA.

\section{Coût d'installation et d'exploitation d'une BFA}

Dans les conditions expérimentales de cette étude, le coût d'installation et d'exploitation d'une BFA de $625 \mathrm{~m}^{2}$ a été évalué à 896188 FCFA (environ $1350 €$ ) (tableau IV). Les coûts d'installation de la clôture, de la production des plants, du transport des plants et de la main-d'œuvre pour la plantation ont été élevés parce que nous avons fait appel à des prestataires extérieurs (entrepreneur en bâtiments, pépiniériste, main-d'œuvre non familiale) auxquels il a fallu payer les services, mais qui ont garanti un travail de qualité, ce qui 
a permis d'optimiser les chances de réussite de cette expérimentation. L'éleveur a apporté le terrain, en a assuré sa préparation (labour, buttage, apport de paille et de fumure), a réalisé l'entretien des BFA (désherbage, fourniture de fumure organique, etc.) et a effectué les coupes de fourrages pour les animaux, conformément aux cahiers des charges.

Ainsi, si nous considérons une durée d'exploitation de la BFA de 10 années et une production moyenne de $5 \mathrm{t} \mathrm{MS}$.ha $^{-1}$ par BFA $(L$. leucocephala et $M$. alba), le coût de production d'un kilogramme de
MS de fourrage d'une BFA s'élève à environ 287 FCFA.kg MS ${ }^{-1}$ (soit $0,43 €)$. Ce coût est bien supérieur au prix des aliments bétail locaux, donc trop élevé et non économiquement viable. Mais, il s'agit ici des conditions d'expérimentation, nous verrons dans la discussion que les économies d'échelle permise par une BFA de plus grande dimension (1 ha) cumulée aux économies possibles avec une BFA réalisée par l'éleveur lui-même abaissent fortement ce coût de production, notamment si les rendements de production de la BFA dépassent 5 t MS. ha ${ }^{-1}$, ce qui est un objectif tout à fait envisageable.

\section{Tableau III}

Valeur nutritionnelle à différentes périodes de Leucaena leucocephala et Morus alba dans les banques fourragères arbustives (BFA) de deux localités de l'ouest du Burkina Faso

\begin{tabular}{|c|c|c|c|c|c|c|c|}
\hline \multirow[b]{2}{*}{ BFA } & \multirow[b]{2}{*}{ Période } & \multicolumn{3}{|c|}{ Leucaena leucocephala } & \multicolumn{3}{|c|}{ Morus alba } \\
\hline & & MAT & NDF & $\mathrm{ADF}$ & MAT & NDF & ADF \\
\hline \multirow[t]{4}{*}{ BFA2 } & Juil. (jp+13 mois) & 28,14 & 41,33 & 22,12 & 19,09 & 35,13 & 28,72 \\
\hline & Sept. (jp+15 mois) & 21,94 & 39,46 & 26,73 & 14,12 & 33,01 & 21,78 \\
\hline & Nov. (jp+17 mois) & $12,21^{\mathrm{a}}$ & $43,21^{\mathrm{a}}$ & $28,96^{\mathrm{a}}$ & $11,52^{\mathrm{a}}$ & $38,73^{\mathrm{a}}$ & $24,44^{\mathrm{a}}$ \\
\hline & Déc. (jp+18 mois) & $11,30^{\mathrm{a}}$ & $41,03^{\mathrm{a}}$ & $26,89^{\mathrm{a}}$ & $10,35^{\mathrm{a}}$ & $41,73^{\mathrm{a}}$ & $27,75^{\mathrm{a}}$ \\
\hline \multirow[t]{4}{*}{ BFA3 } & Juil. (jp+13 mois) & AbsD & AbsD & AbsD & AbsD & AbsD & AbsD \\
\hline & Sept. (jp+15 mois) & AbsD & AbsD & AbsD & AbsD & AbsD & AbsD \\
\hline & Nov. (jp+17 mois) & $12,23^{\mathrm{a}}$ & $45,08^{\mathrm{a}}$ & $30,95^{\mathrm{a}}$ & $9,34^{\mathrm{a}}$ & $36,95^{\mathrm{a}}$ & $24,33^{\mathrm{a}}$ \\
\hline & Déc. (jp+18 mois) & $10,54^{\mathrm{a}}$ & $44,84^{\mathrm{a}}$ & $31,11^{\mathrm{a}}$ & $9,17^{\mathrm{a}}$ & $46,50^{\mathrm{a}}$ & $32,61^{\mathrm{a}}$ \\
\hline
\end{tabular}

MAT : matière azotée totale ; NDF : fibres insolubles dans les détergents neutres ; ADF : fibres insolubles dans les détergents acides ; AbsD : absence de données ; jp : jour de plantation; ${ }^{\text {a }}$ Les valeurs suivies de la même lettre sur la même colonne ne sont pas significativement différentes au seuil de $5 \%$.

Tableau IV

Coût d'installation (FCFA) d'une banque fourragère arbustive (BFA) à I'ouest du Burkina Faso

BFA réalisée dans les conditions de l'étude

Prix unité

Installation clôture en grillage

Ursus renforcée de poteaux

en bois $(m)$

Achat des graines (kg)

Production des plants chez un pépiniériste (plants)

Transport des plants (forfait)

Labour ou buttage (ha)

Désherbage (jours)

Plantation/main-d'œuvre (forfait)

Coût total d'installation

Coût annuel d'entretien et

d'exploitation (coupe)

Coût total (1) pendant 10 ans

Coût total / ha

Coût total / ha / an

Coût total / ha / an / kg MS (2)
$625 \mathrm{~m}^{2}(25 \times 25)$

1 ha $\left(100 \times 100 \mathrm{~m}^{2}\right)$

Quantité Total

31600

1975

256750

50000

938

4500

15000

1500

0,0625

3

30000

851188

4500

1500

3

896188

14339000

1433900

287

400 * $\quad 2000000$

6,4

144000

4108000

120000

BFA « faite maison »

1 ha

Total

1750000

100000

$\begin{array}{crr}16 & 800000 & 0 \\ 1 & 15000 & 15000 \\ 48 & 72000 & 50000 \\ 16 & 480000 & 250000 \\ & 7619000 & 2285000 \\ & 71885 & 50000\end{array}$

50000

$1 €=655,956$ FCFA ; (1) Installation + entretien + exploitation ; (2) Pour un rendement théorique moyen de la BFA de 5 t MS/ha/an

* Le périmètre d'une parcelle de $650 \mathrm{~m}^{2}$ est de $100 \mathrm{~m}$. Le périmètre d'un hectare est de 400 mètres. Les autres postes sont proportionnels à la surface. 
DISCUSSION

\section{Comportement des BFA face aux contraintes pédoclimatiques, aux attaques de termites et au feu}

\section{Influence de la qualité du sol sur le développement des BFA}

Les faibles teneurs en $\mathrm{MO}$ et en phosphore assimilable des sols des BFA pourraient expliquer la faible performance de croissance et de production des plants de L. leucocephala et M. alba par rapport aux résultats rapportés à Cuba (González-García et Martín-Martín, 2016 ; González-García et al., 2009). De plus, l'acidité du sol de la BFA3 $(\mathrm{pH}=5,3)$, par rapport au sol de la BFA2 $(\mathrm{pH}=6,1)$, expliquerait en partie la faible croissance des plants sur la BFA3, surtout celle de L. leucocephala qui supporterait mal les sols à $\mathrm{pH}$ acide (Bertrand, 2009).

\section{Effet du stress hydrique sur le développement des BFA}

A jp+7 mois, le développement des BFA a été similaire, voire supérieur à ceux rapportés à Cuba (Wencomo et Ortiz, 2009 ; Pentón et al., 2007 ; Noda et Martín, 2008). Cependant, à partir de jp+5 jusqu'à jp+7 mois, en l'absence des pluies, les plants ont été soumis à un stress hydrique important ; cela s'est traduit par le ralentissement de leur développement qui s'est quasi arrêté en saison sèche chaude. Le stress hydrique a également provoqué le fanage des tiges tendres ou des bourgeons apicaux des plants des deux espèces, mais avec un effet plus marqué sur M. alba, sans pour autant entraîner des mortalités.

\section{Effet de l'attaque de termites sur les BFA}

L'effet combiné des facteurs sol et stress hydrique ont entraîné une fragilisation de l'état des plantes en les rendant vulnérables aux termites en saison sèche (Jouquet et al., 2018). Très peu d'espèces de termites s'attaquent aux cultures en bonne santé (Louppe et Zaremski, 2016). Les termites préfèrent du matériel végétal desséché. Or dans la BFA3, en l'absence de paillis pour fournir de la matière organique aux termites, ceux-ci se sont rabattus sur les plants de M. alba qui étaient déjà stressés par un arrêt précoce des pluies sur ce site. Selon la méthode dite « alimentaire », décrite par Han et Ndiaye (1996), qui consiste à fournir aux termites du bois mort pour les détourner des plantes cultivées, le paillis aurait pu jouer un rôle de leurre. D'autres méthodes biologiques et mécaniques de lutte existent comme l'utilisation de champignons entomopathogènes, la stimulation des prédateurs naturels tels que les fourmis (Jouquet et al., 2018), l'application d'extraits d'Azadirachta indica (Tahiri et al., 2011), la destruction des nids.

\section{Capacité de régénération des BFA après le passage du feu}

L. leucocephala et M. alba sont caractérisées par un système racinaire pivotant et traçant, capable d'explorer des profondeurs de plus de deux mètres (Datta, 2000 ; CTA, 1987). Cette aptitude des plants, combinée à l'arrosage des plants à l'arrosoir durant les quatre semaines qui ont suivi le feu, ont expliqué la forte régénération des plants de la BFA1 à jp+13 mois. La BFA1 a, à ce jour, récupéré à 100 $\%$ grâce à quelques replantations mais accuse toujours un retard par rapport aux BFA2 et BFA3.

\section{Performances de production prometteuses sur les plans quantitatif et qualitatif, et saisonnalité des BFA}

Les rendements moyens présentés dans cette étude ont été obtenus alors que les BFA étaient encore à l'état juvénile dans leur première année de production. Nous estimons que, comme d'autres études l'ont montré, ces rendements vont augmenter les années suivantes avec la maturité des BFA (Dandin et Sengupta, 1988 ; Tewari et al., 2004), ce qui laisse espérer des rendements optimaux en troisième ou quatrième année d'exploitation.

En revanche, la production des BFA en saison sèche a été faible et n’a représenté que $20 \%$ de la production annuelle de biomasse en saison sèche froide, et $10 \%$ en saison sèche chaude. Produire du fourrage vert de bonne valeur nutritionnelle et en quantité pendant la saison sèche n'est donc pas un objectif facile à atteindre, même avec des fourrages ligneux. Des travaux restent à mener pour identifier des fourrages ligneux plus productifs en saison sèche ou pour rechercher des moyens pour augmenter la production en saison sèche sans augmenter considérablement le coût de production. Sur le plan de la composition chimique, les résultats obtenus (tableau III) sont conformes à ceux de González-García et al. (2009), et de González-García et Martín-Martín (2016) à Cuba, confirmant la bonne qualité nutritionnelle des BFA.

\section{Ajustement du coût de production des BFA à la hauteur des possibilités des éleveurs}

La mise en place d'une BFA par un producteur exige un foncier sécurisé, ce qui n'est pas à la portée de tous les éleveurs. Dans les conditions de l'étude, le coût d'installation et d'exploitation d'une BFA d'un hectare a été de 8337850 FCFA (environ $12700 €$ ) (tableau IV), ce qui est très élevé. Toutefois, l'augmentation de la dimension d'une BFA permet de réaliser une importante économie d'échelle par rapport à une BFA de $625 \mathrm{~m}^{2}$, parce que le coût de la clôture (qui est proportionnel au mètre linéaire) augmente moins vite que les autres coûts (qui sont proportionnels au mètre carré). De plus, si la BFA est réalisée par l'éleveur lui-même, alors d'importantes économies peuvent être réalisées sur plusieurs postes et faire baisser de manière significative son coût d'installation et d'exploitation. En nous basant sur les coûts réels pratiqués par les éleveurs et la qualité du matériel qu'ils utilisent habituellement, nous pouvons estimer le coût d'un hectare de BFA à environ 2785000 FCFA (environ $4250 €$ ) (tableau IV). Il faut arriver à ce que les BFA puissent être une ressource fourragère compétitive par rapport aux principaux compléments disponibles : tourteau de coton 150 à 250 FCFA.kg- $^{-1}(0,23$ à $0,38 €)$, et fane de mucuna 50 FCFA.kg-1 $(0,08 €)$.

\section{Autres propositions techniques pour adapter les BFA aux besoins et possibilités des éleveurs}

Le choix des espèces à installer doit tenir compte de plusieurs facteurs : croissance rapide, rendement élevé, bonne valeur nutritionnelle, et capacité à résister à la sécheresse et à tolérer des sols pauvres. Dans le contexte pédoclimatique de cette étude, il paraît raisonnable d'envisager des BFA avec des espèces introduites ou locales qui semblent plus adaptées (L. leucocephala, G. sepium, A. lebbeck, Pterocarpus erinaceus, Faidherbia albida, entre autres).

Il peut être proposé des ajustements de l'itinéraire technique pour améliorer l'étape d'installation par l'approfondissement des lits de plantation (au minimum $30 \mathrm{~cm} \times 30 \mathrm{~cm}$ ) comme recommandé par Datta (2000), un amendement de fond à l'implantation et à chaque coupe, à raison de 2,5 t MS.ha ${ }^{-1}$ de fumier, un désherbage régulier, un paillage précoce après l'arrêt des pluies à raison de 10 t MS.ha ${ }^{-1}$, précédé d'un apport de fumure et d'un buttage, et l'installation de pare-feu bien entretenus.

Diverses alternatives d'associations des arbres fourragers dans les exploitations existent (Bationo et al., 2012). Toutefois, le choix des BFA à haute densité répond au besoin de produire du fourrage en quantité sur des surfaces restreintes. De plus, des études montrent que dans la limite de 40000 plants.ha $^{-1}$, une densité de plantation élevée est corrélée positivement avec un rendement fourrager élevé, optimum à 20 000-25 000 plant.ha $^{-1}$ (Noda et Martín, 2008). Cette 
technique de production se justifie au Burkina Faso notamment en raison du manque de terres disponibles pour la production fourragère.

Par ailleurs, nous proposons une adaptation du mode d'exploitation des BFA avec deux coupes à 90 jours d'intervalle (première coupe le 15 août et deuxième coupe le 15 novembre) en lieu et place du rythme de récolte de 60-90-120 jours proposé pour les BFA à Cuba par González-García et Martín-Martín (2016). En fonction des besoins, le fourrage coupé en saison des pluies sera utilisé en vert ou stocké par séchage permettant d'augmenter les stocks de réserves fourragères pour la saison sèche.

\section{Coconception : une démarche d'apprentissage et d'adaptation de l'innovation aux besoins de l'éleveur}

Cette étude est née de la volonté des éleveurs d'améliorer l'affourragement de leurs animaux et de la volonté des chercheurs d'accompagner les éleveurs dans la conception d'une innovation dans le système fourrager local. Un partenariat a été matérialisé par l'implication des chercheurs, des éleveurs et des acteurs de développement des différents sites d'études, à travers des ateliers participatifs qui ont permis d'apprendre les principes théoriques de la BFA et d'adapter la technologie de production des BFA au contexte local. Avec la démarche de coconception, les chercheurs ont réalisé un diagnostic des systèmes de production chez des éleveurs laitiers (Sib et al., 2017). Ce diagnostic a permis aux chercheurs et aux éleveurs d'identifier des solutions possibles à expérimenter, et d'élaborer des cahiers des charges pour définir les engagements de chaque partie (Vall et al., 2016).

La coconception a permis d'adapter les pratiques de plantation, de gestion et d'exploitation dans le contexte des fermes laitières agropastorales du Burkina Faso. Elle a montré que le choix d'espèces ligneuses exotiques comme M. alba, exigeant en fertilisants et en eau, n'est pas adapté au contexte des exploitations agropastorales locales caractérisées par des sols pauvres en matière organique et en phosphore.

La coconception a permis, en outre, d'adapter le schéma d'implantation d'une BFA en fonction des contraintes des éleveurs. Ainsi, le choix du terrain en fonction du plan parcellaire et du système cultural de l'éleveur a facilité un suivi rapproché des BFA. La densité a été réajustée par rapport au taux de germination des graines mises en pépinière. En l'absence d'une traction motorisée dans les exploitations, le sillonnage a été réalisé par un attelage bovin, ce qui expliquait des labours et des lits de plantation peu profonds dans certains cas. Ces lits de plantation ont été parfois approfondis manuellement avant les implantations.

Les dispositifs de protection pour faire face à la divagation des bovins, des ovins et des caprins, et pour sécuriser les parcelles expérimentales devaient être adaptés ; les clôtures en grillage ont été renforcées par des poteaux en bois. Les haies vives de G. sepium, d'A. lebbeck et de $S$. saman ont été renforcées par des plants d'Acacia nilotica, bien connus pour constituer des haies vives défensives efficaces (Bationo et al., 2012). Pour protéger les plants des feux de brousse récurrents, des pare-feu ont été créés autour des BFA mais le manque d'entretien a entraîné le feu dans la BFA1. Le paillage a eu pour objectif premier de protéger les racines des plantes contre la sécheresse ; il s'est toutefois révélé efficace dans la lutte contre les termites dans la BFA2.

Les techniques de mise en place d'une BFA ont été adaptées progressivement pendant l'étape d'installation. En première année d'expérimentation (2016), les éleveurs n'étaient pas familiarisés aux techniques de production de plants en pépinières, ce qui nous a amenés à utiliser les services d'un pépiniériste professionnel à Bobo-Dioulasso. En 2017, les éleveurs ont été formés à la production des plants en pépinière dans leur exploitation, ce qui a permis de réduire le coût d'installation. L'implantation des deux espèces en deux blocs répondait au besoin de suivre séparément les deux espèces sur la même parcelle afin de collecter des références en cultures pures. D'après les cahiers des charges élaborés avec les chercheurs, les éleveurs expérimentateurs ont apporté du fumier des parcs pour la fertilisation des parcelles et désherbé régulièrement les BFA pour éviter la compétition avec les mauvaises herbes.

Enfin la démarche de coconception a permis de proposer des plans d'exploitation compatibles avec une exploitation durable de la BFA au regard des conditions pédoclimatiques. En effet, la saison des pluies dure au plus cinq mois, de juin à octobre, pour une gestion durable il faudra éviter de stresser les plantes pendant la période sèche afin de leur permettre de réaliser leur photosynthèse en attendant la saison des pluies prochaine. De même, les éleveurs ont reçu à la demande une formation sur les méthodes de conservation des fourrages récoltés en saison des pluies, ce qui a permis de limiter le gaspillage du fourrage vert.

\section{CONCLUSION}

Ce travail a montré la capacité des banques fourragères arbustives à haute densité à s'installer dans les conditions de l'ouest du Burkina Faso pour compléter l'alimentation du bétail.Leucaena leucocephala et dans une moindre mesure Morus alba ont affiché une bonne adaptation à l'environnement local, caractérisé par des sols pauvres et des conditions climatiques fluctuantes et par la présence de termites ravageurs.

Pour parvenir à une bonne installation et augmenter les rendements des BFA, le choix des espèces ligneuses et l'optimisation des pratiques agronomiques sont indispensables pour réduire les effets de la pauvreté des sols et de la variabilité climatique. La BFA pourrait être utilisée en substitution partielle aux aliments concentrés en saison sèche avec conservation de la coupe de saison des pluies et l'affourragement à l'auge pour la coupe de saison sèche. Des efforts devront se poursuivre afin de trouver des solutions pour réduire le coût d'installation et d'exploitation des BFA afin de rendre accessible l'innovation à un plus grand nombre d'éleveurs.

\section{Déclaration des contributions des auteurs}

OS, EV, EGG, MB et VMCBY ont participé à la conception et à la planification de l'étude ; OS a recueilli les données ; OS et EV ont analysé, interprété les données et rédigé la première version du manuscrit ; OS, EV, EGG ont participé à la révision du manuscrit.

\section{Conflits d'intérêt}

Les auteurs déclarent ne pas avoir de conflit d’intérêts.

\section{REFERENCES}

Bationo B.A., Kalinganire A., Bayala J., 2012. Potentialités des ligneux dans la pratique de l'agriculture de conservation dans les zones arides et semiarides de l'Afrique de l'Ouest : aperçu de quelques systèmes candidats. World Agroforestry (ICRAF) Nairobi, Kenya (Technical Manual; 17)

Bertrand G., 2009. Caractérisation des réponses adaptatives à la contrainte hydrique dans le sud-est de l'Amazonie chez trois espèces fourragères cultivées en monoculture et en association : Brachiaria brizantha, Leucaena leucocephala et Arachis pintoi. Thèse Doct., Université Paris-Est, France

Chave J., Rejou-Mechain M., Burquez A., Chidumayo E., Colgan M.S., Delitti W.B.C., Duque A., et al., 2015. Improved allometric models to estimate the aboveground biomass of tropical trees. Global Change Biol., 20: 3177 3190, doi: 10.1111/gcb.12629

Corniaux C., Duteurtre G., Broutin C., 2014. Filières laitières et développement de l'élevage en Afrique de l'Ouest. L'essor des minilaiteries. Karthala, Paris, France, 252 p. (Coll. Homme et sociétés) 
CTA, 1987. Leucaena, I'arbre champion toutes catégories. Spore (8)

Dandin S.B., Sengupta K., 1988. Mulberry cultivation as high bush and small trees in hilly regions. Central Sericultural Research and Training Institute, Srirampura, Mysore, India, $24 \mathrm{p}$.

Datta R.K., 2000. Mulberry cultivation and utilization in India. In: FAO E-Conf. Mulberry for animal production (Morus-L). FAO, Rome, Italy

Franzel S., Carsan S., Lukuyu B., Sinja J., Wambugu C., 2014. Fodder trees for improving livestock productivity and smallholder livelihoods in Africa. Curr. Opin. Environ. Sustain., 6: 98-103

González-García E., Cáceres O., Archimède H., Santana H., 2009. Nutritive value of edible forage from two Leucaena leucocephala cultivars with different growth habits and morphology. Agrofor. Syst., 77: 131-141, doi: 10.1007/s10457-008-9188-4

González-García E., Martín-Martín G., 2016. Biomass yield and nutrient content of a tropical mulberry forage bank: effects of season, harvest frequency and fertilization rate. Grass Forage Sci., 72: 248-260, doi: $10.1111 /$ gfs. 12227

Han S.H., Ndiaye A.B., 1996. Dégâts causés par les termites (Isoptera) sur les arbres fruitiers dans la région de Dakar (Sénégal). Actes Collect. Insectes Soc., 10 : 111-117

Jouquet P., Chaudhary E., Kumar A.R.V., 2018. Sustainable use of termite activity in agro-ecosystems with reference to earthworms. A review. Agron. Sustain. Dev., 38: 3, doi: 10.1007/s13593-017-0483-1

Klein H.-D., Rippstein G., Huguenin J., Toutain B., Guerin H., Louppe D., 2014. Les cultures fourragères. Quae, Versailles, France (Coll. Agricultures tropicales en poche ; 12), doi : 10.35690/978-2-7592-2169-1
Noda Y., Martín G., 2008. Efecto de la densidad de siembra en el establecimiento de morera para su inclusión en sistemas ganaderos. Zootec. Trop., 26 (3): 339-341

Louppe D., Zaremski A., 2016. Les termites. In : Les rendez-vous de la science, Muséum d'histoire naturelle, Nantes, France, 18 p. https:// agritrop.cirad.fr/580482/9/580482.pdf

Pentón G., Martín G., Pérez A., Noda Y., 2007. Comportamiento morfoagronómico de variedades de morera (Morus alba L.) durante el establecimiento. Morphoagronomic performance of mulberry (Morus alba L.) varieties during the establishment. Pastos Forrajes, 30 (3): 315-325

Sib O., Bougouma-Yameogo V.M.C., Blanchard M., Gonzalez-Garcia E., Vall E., 2017. Dairy production in Western Burkina Faso in a context of emergence of dairies: Diversity of breeding practices and proposals for improvement. Rev. Elev. Med. Vet. Pays Trop., 70 (3): 81-91, doi: 10.19182/remvt.31521

Tahiri A., Amissa Adima A., Adje A.F., Amusant N., 2011. Effet pesticide et screening des extraits de Azadirachta indica (A.) Juss., sur le termite Macrotermes bellicosus Rambur. Bois For. Trop., 310, 79-88, doi : 10.19182/bft2011.310.a20461

Tewari S.K., Katiyar R.S., Balak Ram, Misra P.N., 2004. Effect of age and season of harvesting on the growth, coppicing characteristics and biomass productivity of Leucaena leucocephala and Vitex negundo. Biomass. Bioenergy, 26: 229-234, doi: 10.1016/S0961-9534(03)00118-1

Vall E., Chia E., Andrieu N., Blanchard M., Koutou M., Coulibaly K., 2016. La co-conception en partenariat de systèmes agricoles innovants. Cah. Agric., 25, 15001, doi : 10.1051/cagri/2016001

Wencomo H.B., Ortiz R., 2009. Comportamiento de 23 accesiones de Leucaena spp. en condiciones de establecimiento. Pastos Forrajes, 33 (3)

\section{Resumen}

Sib O., González-García E., Bougouma-Yameogo V.M.C., Blanchard M., Vall E. Co-concepción, instalación y evaluación de bancos de forrajeras arbustivas para la alimentación de vacas lecheras en el oeste de Burkina Faso

En las fincas lecheras del oeste de Burkina Faso, la producción de leche está limitada por un déficit forrajero durante la estación seca. Este estudio pretendió evaluar la factibilidad y la potencialidad de los bancos de forrajeras arbustivas (BFA) para complementar la alimentación de las vacas lecheras durante la estación seca con un forraje de buen valor nutricional. Se llevó a cabo un trabajo de co-concepción, de instalación y explotación de BFA compuestos por Leucaena leucocephala y de Morus alba, en tres fincas de bovinos de leche del oeste de Burkina Faso. Los tres BFA se plantaron con densidad alta, con 20000 plantas.ha ${ }^{-1}$, en julio 2016. El crecimiento fue rápido, durante los siete primeros meses (estación de lluvias e inicio de la estación seca). Al día de plantación (dp) más siete meses, L. leucocephala midió $145 \pm 11 \mathrm{~cm}$ y M. alba $143 \pm 72 \mathrm{~cm}$. Seguidamente, durante la estación seca, el crecimiento disminuyó. Las características del suelo, la falta de lluvias y la poca profundidad de los lechos de cultivo explicaron las diferencias de crecimiento observadas según los sitios experimentales. Los ataques de termitas (BFA3) y el paso del fuego (BFA1) afectaron el desarrollo de los BFA concernidos sin aniquilarlos. La producción de biomasa en BFA2 y BFA3, acumulada en las tres podas de explotación (dp+13, dp+15 y dp+17-18 meses),

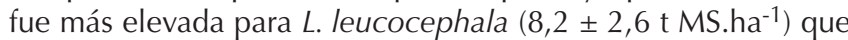
para $M$. alba $\left(1,8 \pm 2,3\right.$ t MS.ha $\left.^{-1}\right)$. El costo de instalación y explotación de $625 \mathrm{~m}^{2}$ de BFA fue de 896188 FCFA (aproximadamente $1350 €$ ). Deben encontrarse soluciones para hacerlas financieramente más accesibles a los productores.

Palabras clave: ganado de leche, forrajes, plantas de ramoneo, experimentación en campo, alimentación de rumiantes, Burkina Faso 
